\title{
CAE Analysis for Oil Leakage Mechanism of Transaxle Oil Seal
}

\author{
Ryo Akaiwa ${ }^{1}$, Kakuro Amasaka ${ }^{2}$ \\ ${ }^{1}$ (Management Technology, Aoyama Gakuin University, Japan) \\ ${ }^{2}$ (Management Technology, Aoyama Gakuin University, Japan)
}

\begin{abstract}
In recent years, the authors have been conducting research on the establishment of technical prevention measures for automobile development and design. This research investigates the mechanisms that cause oil leaks in drive train oil seals, which are a matter of concern for automobile manufacturers around the world. In the case of automobile transaxle oil seals, the edge of the seal's rubber lip is in sliding contact with the surface of the drive shaft, and the seal serves to prevent the lubricating oil sealed inside the drive gearbox from leaking to the outside. In this paper, the authors take up the example of analyzing the mechanisms by which oil leaks caused by wear of drive train oil seals. In order to demonstrate this, the authors incorporated a model of the oil film on sliding surfaces and simulated the oil seals' sealing performance. The authors also incorporated the analysis model for CAE, combining a fluid model, an oil seal sliding surface model, and methods such as the finite element method. Thus, the authors contributed to establishing technical prevention measures for automobile development and design utilizing CAE.
\end{abstract}

Keywords: Computer Aided Engineering, Finite Element Method, Oil Seal

\section{Introduction}

The Japanese automobile industry is urgently seeking ways to reduce development and design periods and achieve improvement of Quality, Cost, and Delivery (QCD) at the same time. [1,2] In order to realize them, Computer Aided Engineering (CAE) plays an important role. [3] However, in the design of parts such as oil seals, quality improvement is slow in relation to the reduction of development periods. One of the reasons is believed to be that mechanisms of parts were not incorporated into CAE [4, 5].

In this research we applied "technical element model for high reliable CAE analysis" to understand the relationship of each technical elements. It is constructed with "Problem" and "Numerical Expression", "CADdata", "Algorithm", "Interpretation". We tried to find a main cause of oil leaking by an affinity diagram. Then we applied the basic sliding surface structural model $[6,7]$ to the CAD-data. For this we used a concept of oil film formed between oil seal and shaft [8-10]. Finally we simulated the sealing performance of oil seal by using Finite Element Method (FEM) as an algorithm.

\section{CAE Analysis Model for Oil Seal}

2.1 Understanding oil leaking phenomena

When examining the oil seal leak problem, the cause-and-effect relationships of the factors must be grasped by referring to an affinity diagram, as shown in Fig. 1. In our research, we found six categories: pumping function, foreign matter, usage environment, wear, shaft, and installation/custody. We grouped the abovementioned six items into "oil seal design stage" and "oil seal usage stage."

The design stage group included pumping function, foreign matter and wear. Pumping function retains lubricating oil by means of the suction phenomenon resulting from the surface irregularities on the sliding contact of the oil seal as shown in Fg.2 [6]. If foreign matter enters and adheres to the area between the oil seal and shaft, cavitation occur in the oil film formed between oil seal and shaft, and this causes scratches on the seal lip [9]. For the research on pumping function and foreign matter, numerical simulation is presently utilized [8]. However, there has been no simulation for the examination of wear. In light of this, we conducted research on the effect of wear on the sealing performance.

\subsection{Studying numerical expressions of oil leak factors}

In order to simulate the effect of wear on the sealing performance, it is necessary to examine how to express wear numerically. Thus, we decided to adopt the Basic Sliding Surface Structural Model [6]. The Basic Sliding Surface Structural Model approximates the microscopic roughness of sliding surface. 


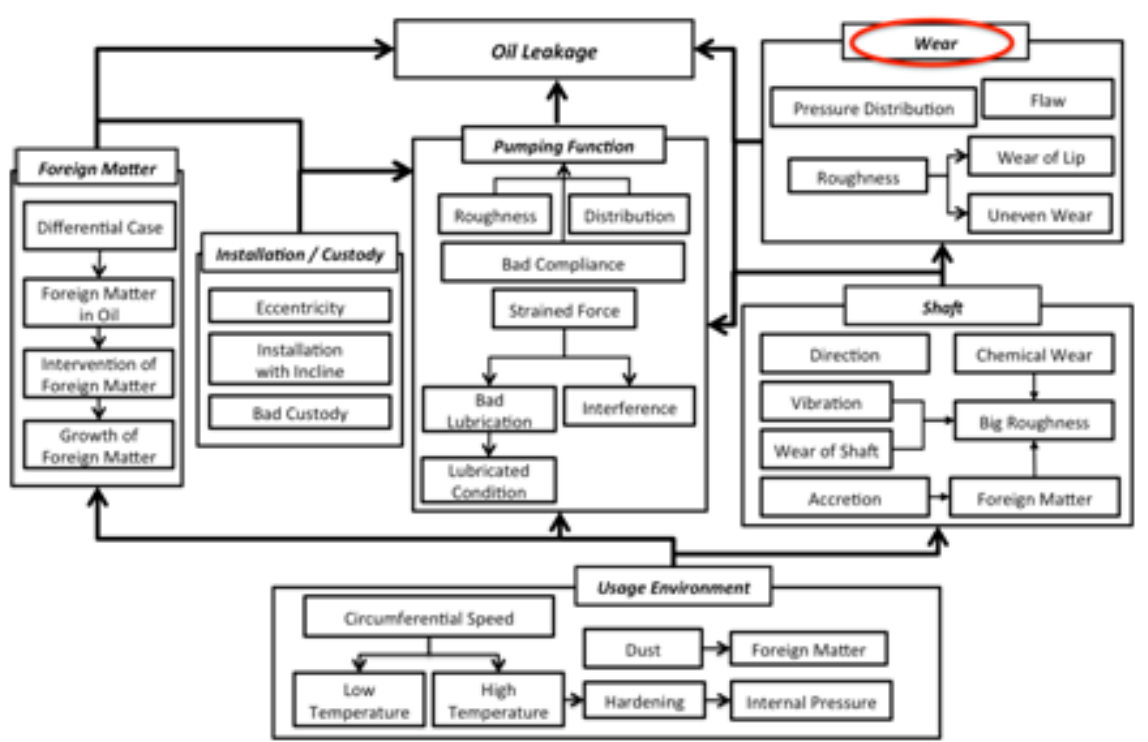

Fig.1 affinity diagram

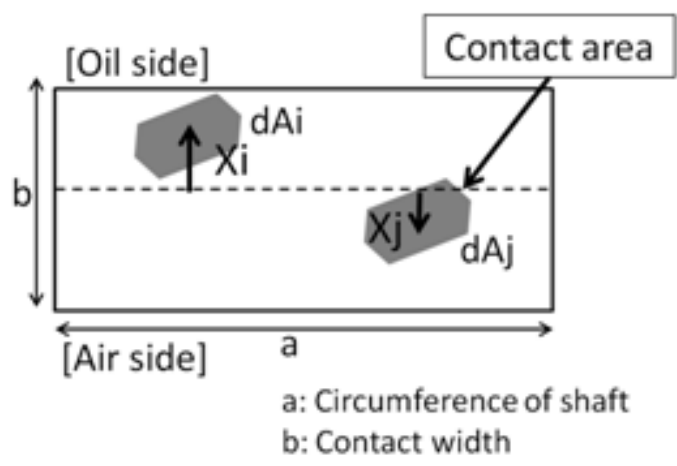

Fig. 2 contact area on sliding surface

$($ Rate of contact $)=($ Contact area $) /($ Sliding surface $)=($ Shaded area $) /\left(a^{*} b\right)$

By understanding those oil flows, it is possible to evaluate the sealing performance. If the amount of oil flow to the oil side is greater than that of into the atmosphere, the oil seal is providing sufficient sealing performance [6]. The Basic Sliding Surface Structural Model can be expressed with two parameters as shown in formulae (1), (2) [6]. Rate of contact is rate of actual contact between oil seal and shaft. Contact deviation is the degree how the center of gravity of the contact surface toward the oil side deviate. To incorporate this model, oil film formed between oil seal and shaft is desirable to be selected as the analysis model (CAD-data) [8]. There are two reasons for this. One is the shape of oil film is affected by the sliding surface condition, and oil leaks from the space between oil seal and shaft.

\subsection{CAE Analysis Model for Oil Seal}

As shown in Fig 3, we defined details of our examination of sealing performance analysis by applying CAE analysis model. We correlated Problem, Numerical Expressions, CAD-data, Algorithm and Interpretation so that we could produce successful analysis results. Based on the affinity diagram shown in Section 3.2, we found that wear is one of the major effects of oil leak. So we set the effect of wear on oil leaks as (i) Problem. Then we used the Basic Sliding Surface Structural Model explained [6] in Section 3.3 as (ii) Numerical expressions to express the problem numerically. In order to reflect the Numerical Expression we designed the oil film between oil seal and shaft as (iii) CAD-data [8]. As (iv) Algorithm we adopted Finite Element Method (FEM). FEM imposes no restriction on the shape of the computational region [4]. Since the oil film model has complicated shape, FEM is suitable for use in this research.

Then we have to consider how to evaluate the sealing performance. There is oil flowing to inside (inlet) and outside (outlet) [6]. It is known that if inlet minus outlet is greater than zero, sealing performance is 
successful $[6,8]$. Therefore, when the inlet was larger than outlet, we determined that the oil seal offers sufficient sealing performance. We configured an oil seal simulator using the abovementioned elements. Successful analysis results depend on the relevance of technical elements.

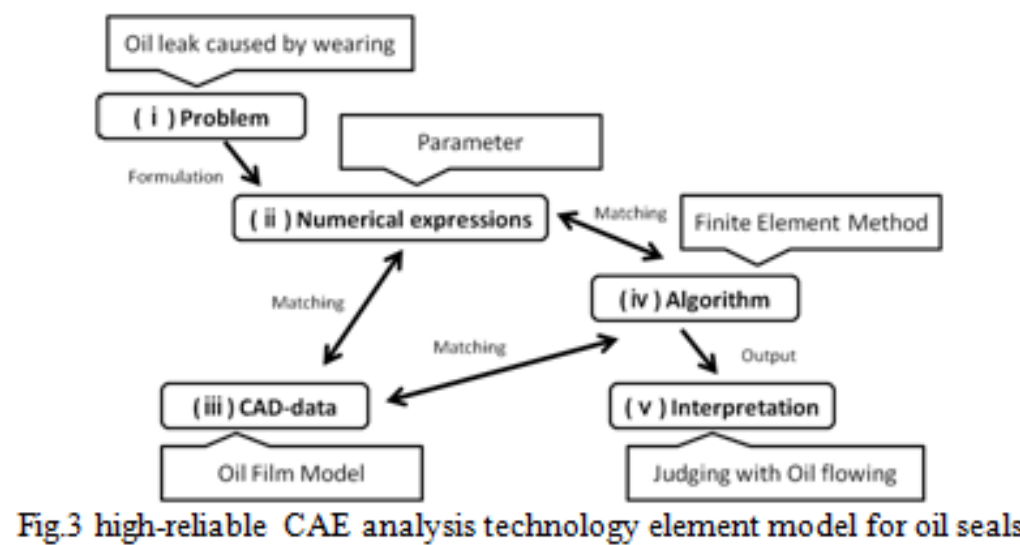

\section{Simulating Sealing Performance}

We incorporated the expression method discussed in Section 2 into the simulation model, and loaded it into the fluid analysis software.

\subsection{Three patterns subject to analysis}

We conducted simulation of three different patterns of the sliding surface condition as shown in Table 1 [6]. In Pattern 1, the rate of contact between the oil seal and shaft was $2 \%$, and the contact deviation was 0.14 . In Pattern 2, the rate of contact was $47 \%$, and the contact deviation was 0.05 . In Pattern 3, the rate of contact was $4 \%$, and the contact deviation was -0.21 .

Table 1 Three patterns subject to analysis

\begin{tabular}{llll}
\hline & Pattern 1 & Pattern 2 & Pattern 3 \\
\hline Rate of contact & 0.02 & 0.47 & 0.04 \\
Contact deviation & 0.14 & 0.05 & -0.21 \\
\hline
\end{tabular}

\subsection{Simulating sealing performance}

Fig 4 shows the contour diagrams of the oil flow on the atmosphere-side. A contour diagram indicates the size distribution of output values using color. In a contour diagram, the larger the value is, the closer the indicated color is toward the red end of the color spectrum. The smaller the value is, the closer the indicated color is toward the blue end of the color spectrum. We compared the total amount of outflow oil on the atmosphere side and the total amount of inflow oil on the oil side.

If the value obtained by subtracting the total amount of outflow oil on the atmosphere side from the total amount of inflow oil is greater than zero, the sliding surface can be said to provide sufficient sealing performance. We used the computation function of ANSYS CFX to calculate the total amount of oil that flows across the plane surface, and then output the abovementioned values. The following shows the result of calculation for Pattern 1 (Unit: $10^{-5}\left[\mathrm{~mm}^{3} / \mathrm{s}\right]$ ) as shown in Table 2 .

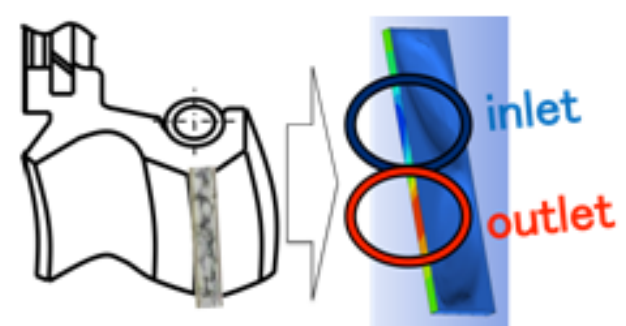

Fig.4 the contour diagrams of the oil flow (Pattem 1) 


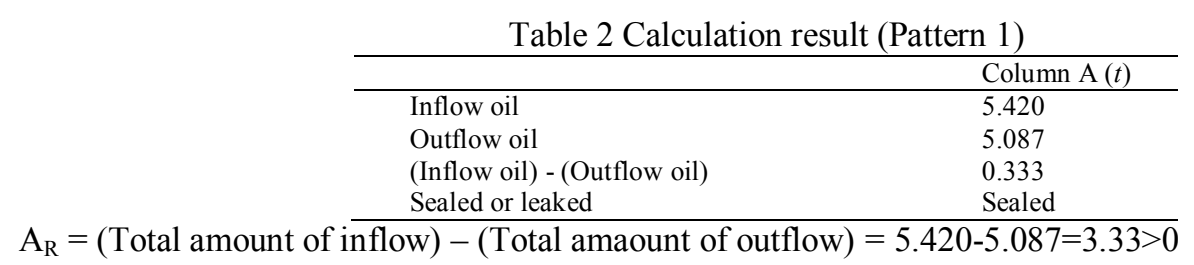

The above calculation result indicates that the oil seal has sufficient sealing performance. The numerical analysis explained above was also conducted for the other two patterns. The next step is verification.

\subsection{Verification}

As shown in Fig. 5 and Table 3, we compared the analysis results with the real machine test. This comparison result verified that the simulation was capable of reproducing sealing performance. "Wear" was expressed as expansion of the real contact region. The review of research results indicated that oil leaks could occur when the contact region became $10 \%$ of the entire sliding surface. Furthermore, close examination of the analysis results revealed that there was a difference in oil flow condition on the atmosphere side between Pattern 1, in which sealing performance was confirmed, and Pattern 2 and Pattern 3, in which oil leaks occurred. In the patterns in which oil leaks occurred, the conditions of oil inflow and outflow were clear. In those cases, there was evidence of a suction-induced flow immediately next to the leaking location. We believe that this phenomenon was attributable to the shift of the oil inflow-outflow balance toward the atmosphere side.

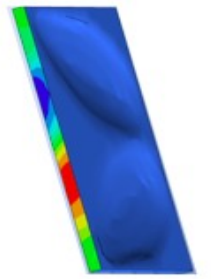

Pattern 1

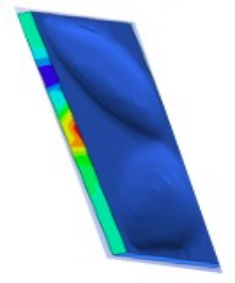

Pattern 2

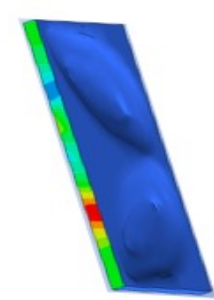

Pattern 3

Fig. 5 the contour diagrams of the oil flow

Table 3 Result of CAE test

\begin{tabular}{llll}
\hline & Pattern 1 & Pattern 2 & Pattern 3 \\
\hline Inlet Flow & 5.42 & 1.99 & 7.67 \\
Outlet Flow & 5.09 & 2.22 & 8.38 \\
(Inlet Flow) - (Outlet Flow) & 0.333 & -0.229 & -0.710 \\
Sealed or leaked & Sealed & Leaked & Leaked \\
\hline
\end{tabular}

\section{Conclusion}

Today CAE plays an important role as a tool for simultaneous achievement of QCD. It can be said in the case of oil seal. In this research, we tried to find a main cause of oil leaking by an affinity diagram. Then we applied the basic sliding surface structural model to the CAD-data. For this we used a concept of oil film formed between oil seal and shaft. Finally we simulated the sealing performance of oil seal by using FEM as an algorithm. In the future studies we hope to simulate oil seal's deterioration of time.

\section{References}

[1] Amasaka K, Osaki S. A Demonstrative Study on High Reliability of Drive System Design. John Wiley \& Sons, Inc.; 2001.

[2] Magoshi Y, Fujisawa H, Sugiura T. Simulation technology applied to vehicle development. Journal of Society of Automotive Engineers of Japan; 2003, Vol. 57, No.3, 95-100

[3] Amasaka K. An Integrated Intelligence Development Design CAE Model Utilizing New JIT, Application to Automotive High Reliability Assurance. Journal of Advanced Manufacturing Systems; 2008, Vo.7, No.2, pp.221-241

[4] Amasaka K, Tsubaki H, Yamaji M, Ueno T. Establishment of Bolt Tightening Simulation System for Automotive Industry Application of the Highly Reliable CAE Model. International Business \& Economics Research Journal; 2009, Vol.8, No.5, pp.5767

[5] Mitsuhashi T, Takeoka S, Tanabe T, Yamaji K, Amasaka K. Concept of high reliability CAE for a fixed bolt (Part 2). Journal of the Japanese Society for Quality Control, The 83rd technical conference, Tokyo, Japan; 2007, 107-110

[6] Nakamura K. Sealing mechanism of rotary shaft lip-type seals. Tribology International; 1987, 20(2), 90-101

[7] Nakamura K, Kawahara Y. Proc. 10 ${ }^{\text {th }}$ Int. Conf. on Fluid Sealing. British Hydromechanics Research Association; 1984, C-187

[8] Ito T, Sato M, Yamaji M, Amasaka K. An analysis of bottleneck technology by using experiments and CAE: Example of the automotive transaxle oil seal leakage. International Business \& Economics Research Journal; 2010, Vol.9, No.12, $123-129$.

[9] Amasaka K, and Nozawa Y. Analyzing Cavitation Caused by Metal Particles in the Transaxle: Application of High Quality Assurance CAE Analysis Model. ; 2012.

[10] Nozawa Y, Yamashita R, Amasaka K. A CAE method of design optimization for quality assurance: Analyzing cavitations caused by metal particles in the transaxle. Proc. of the 2011 International Symposium on System Integration ,Kyoto; 2011 